\title{
High-Grade Mixed Adenoneuroendocrine Carcinoma in the Cecum: A Case Report
}

\author{
Sang Ho Shin, Sae Hee Kim, Sung Hee Jung, Ji Woong Jang, Min Seok Kang, Sang Il Kim, Ji Hye Kim, \\ Jun Ho Lee \\ Department of Internal Medicine, Eulji University Hospital, Eulji University College of Medicine, Daejeon, Korea
}

Gastrointestinal neoplasms with an exocrine and a neuroendocrine component are rare. Such neoplasms are called "mixed adenoneuroendocrine carcinomas" (MANECs) according to the most recent World Health Organization classification of gastrointestinal tract neoplasms. MANECs have no specific findings that distinguish them from pure adenocarcinomas. In addition, the optimal management strategy of MANECs is largely unknown. We describe the case of a 32-year-old man with dizziness and abdominal bloating. A cecal mass was suspected based on an image study done at a local clinic. We evaluated the cecal mass by using colonoscopy, contrast enhanced computed tomography of the abdomen, positron emission tomography-computed tomography, and laboratory studies. The patient underwent a right hemicolectomy and adjuvant chemotherapy. The final histopathological diagnosis was a high-grade MANEC of the ascending colon, tumor stage T3N2M0.

Keywords: Mixed adenoneuroendocrine carcinomas; MANECs

\section{INTRODUCTION}

The presence of a neuroendocrine component in gastrointestinal adenomas/adenocarcinomas or an exocrine component in gastrointestinal neuroendocrine neoplasms is reportedly frequently observed in routine practice. However, mixed exocrine-neuroendocrine tumors are rarely found [1]. According to the recent World Health Organization (WHO) classification, neoplasms with an exocrine and a neuroendocrine component are called "mixed adenoneuroendocrine carcinomas" (MANECs) [2]. We report a rare case of a MANEC that arose in the ascending colon and was not distinguishable from an adenocarcinoma.

\section{CASE REPORT}

A 32-year-old male was referred from a local clinic to our gastro-

Received: May 16, 2016 • Accepted: December 19, 2016

Correspondence to: Sae Hee Kim, M.D.

Department of Internal Medicine, Eulji University Hospital, Eulji University College of Medicine, 95 Dunsanseo-ro, Seo-gu, Daejeon 35233, Korea Tel: +82-42-611-3000, Fax: +82-42-611-3261, E-mail: cozy129@eulji.ac.kr

(C) 2017 The Korean Society of Coloproctology

This is an open-access article distributed under the terms of the Creative Commons Attribution NonCommercial License (http://creativecommons.org/licenses/by-nc/4.0) which permits unrestricted noncommercial use, distribution, and reproduction in any medium, provided the original work is properly cited. intestinal department with complaints of dizziness and abdominal bloating. Despite 2 months of conservative treatment for abdominal discomfort, his condition had worsened. Endoscopy and abdominal sonography performed in the local clinic showed no specific findings and a huge mass in the cecal area, respectively. The cecal mass was evaluated by using abdominal computed tomography (CT), colonoscopy, and laboratory studies on admission to our hospital. Initial laboratory data showed $5.2 \mathrm{~g} / \mathrm{dL}$ of hemoglobin and elevated levels of carcinoembryonic antigen (6.96 $\mathrm{ng} / \mathrm{mL}$ ). Colonoscopy revealed a large fungating mass with a tendency to bleed easily, which interrupted the lumen of the ascending colon (Fig. 1). Contrast enhanced CT of the abdomen showed invagination of the terminal ileum into the ascending colon with an ileocecal mass of $3 \mathrm{~cm}$ in size (Fig. 2A), suggestive of ileocecal intussusception due to ileocecal cancer with multiple metastatic mesenteric lymph nodes (Fig. 2B). Because the histology report based on a biopsy sample was a moderately differentiated adenocarcinoma with no other metastatic lesion noted except for several mesenteric and aortocaval lymph nodes on positron emission tomography-CT (PET/CT), the patient underwent a right hemicolectomy on the 7th day of admission.

Gross inspection of the lesion revealed a polypoid and ulceroinfiltrative growth measuring $8.0 \mathrm{~cm} \times 5.5 \mathrm{~cm}$ in the cecum (Fig. 3). The mesocolon was macroscopically intact. The tumor involvement was approximately $70 \%$ of the circumference. The tumor 


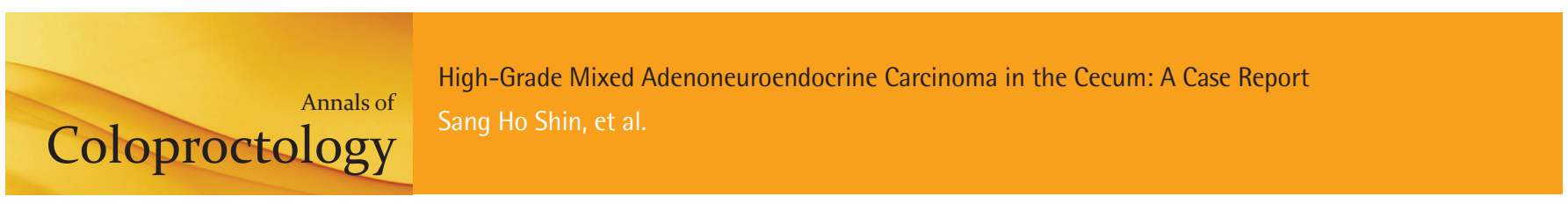

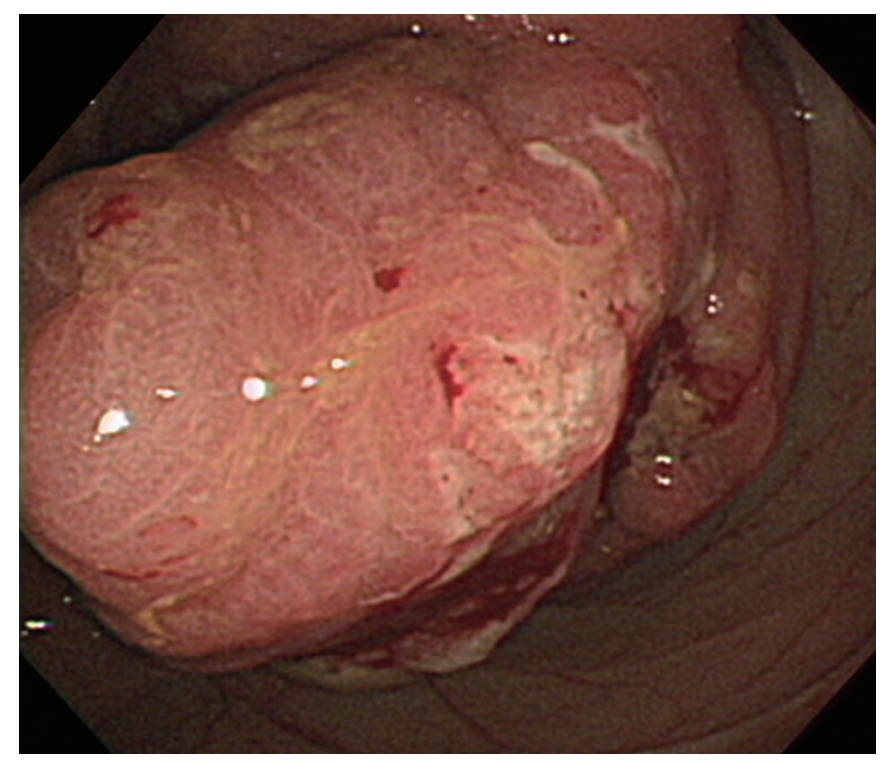

Fig. 1. Colonoscopic finding showed a large fungating mass that interrupts the lumen of the ascending colon.

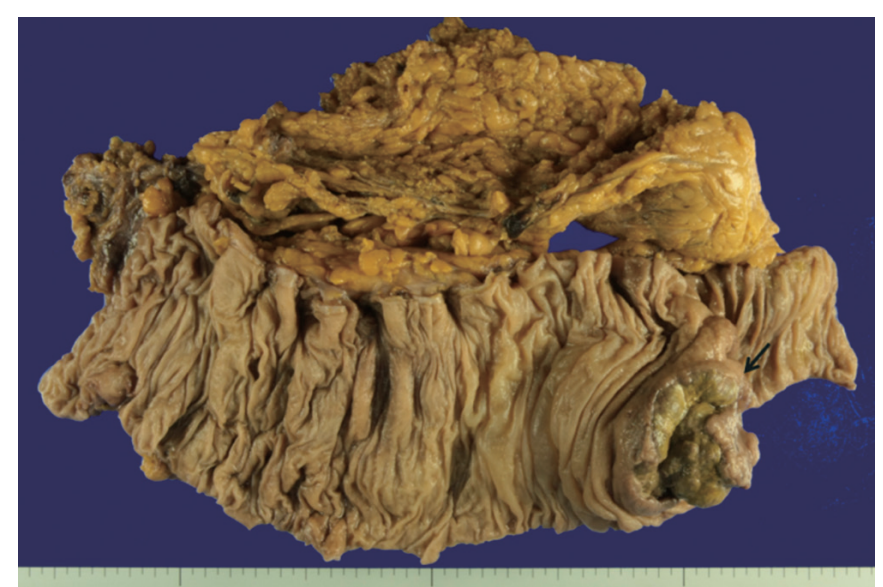

Fig. 3. Gross findings revealed a polypoid and ulceroinfiltrative mass measuring $8.0 \mathrm{~cm} \times 5.5 \mathrm{~cm}$ in the cecum.

invasion depth was to the subserosa. The proximal and the distal resection margins of the specimen were tumor free. Eleven of 49 lymph nodes were metastatic. Sheets of monomorphic large cells with large rounded nuclei and abundant cytoplasm were found on histopathologic examination of the cecal mass. Furthermore, islands of tumor cells with glandular structures accounted for more than $30 \%$ of the tumor (Fig. 4). Immunohistochemical examination revealed synaptophysin (strongly positive) and reactivity in the neuroendocrine differentiation area (Fig. 5). The final histopathological diagnosis was a high-grade MANEC of the ascending colon, tumor stage T3N2M0.

The patient underwent adjuvant chemotherapy. Adjuvant chemotherapy with 5FU and oxaliplatin (FOLFOX) was planned for
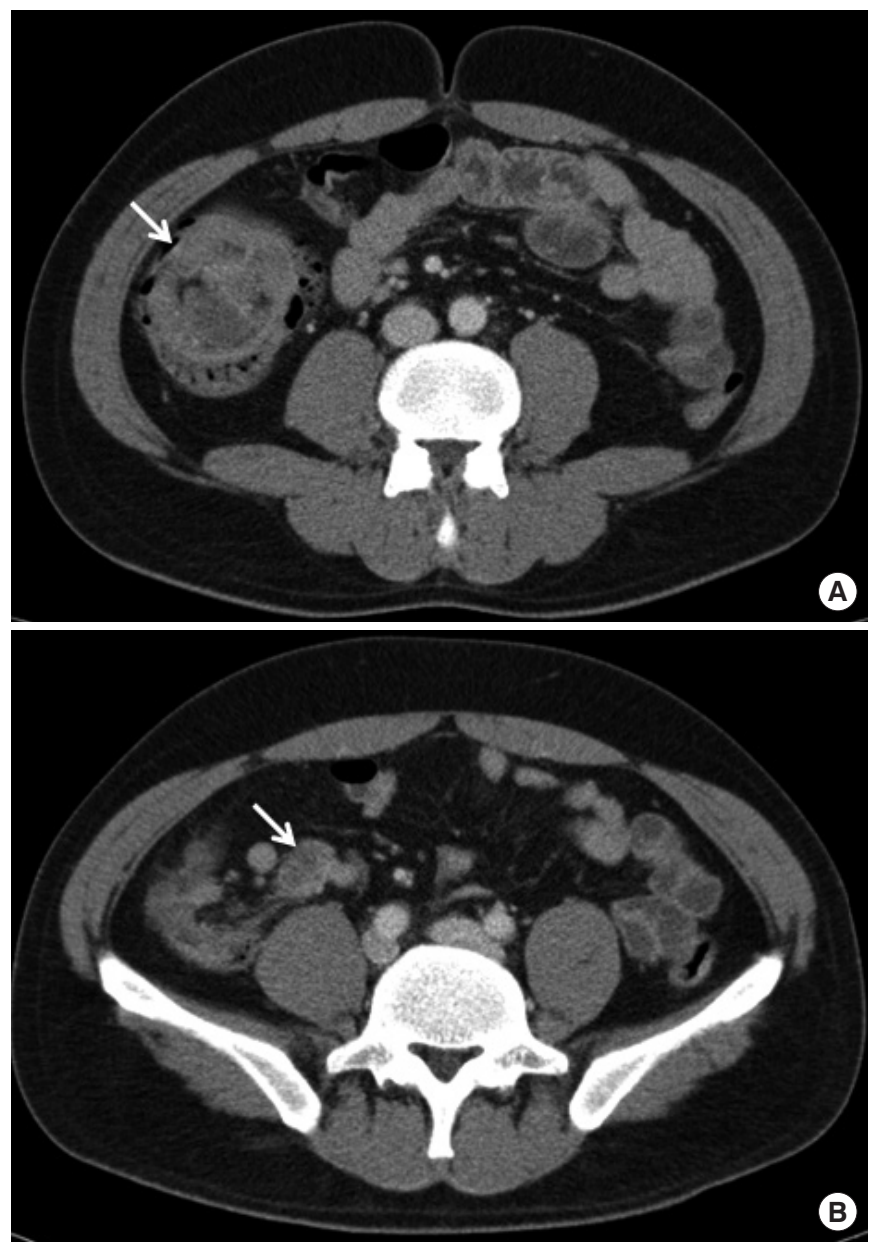

Fig. 2. Contrast-enhanced computed tomography showed invagination of the terminal ileum into the ascending colon with an approximately $3 \mathrm{~cm}$ mass (A; arrow) and irregularly shaped multiple mesenteric lymph nodes with central low density (B; arrow).

six months, and the patient has undergone eight rounds of chemotherapy. The follow-up abdominal CT after four rounds of chemotherapy showed no evidence of tumor recurrence.

\section{DISCUSSION}

A description of exocrine-neuroendocrine tumors of the gastrointestinal tract was first published in 1924 [1]. Early reports since then, include several tumors with various names, such as composite carcinoid, mucin-producing carcinoid, argentaffin cell adenocarcinoma, goblet-cell carcinoid, adenocarcinoid, and smallcell undifferentiated carcinoma [3]. After Lewin's suggested classification of the neoplasm into 3 different subtypes, i.e., collision, combined, and amphicrine tumors, in 1987, the term "mixed tumor" was widely adopted [4]. In accordance with the $2000 \mathrm{WHO}$ classification of endocrine tumors, such neoplasms were defined as mixed exocrine-endocrine tumors when each component rep- 


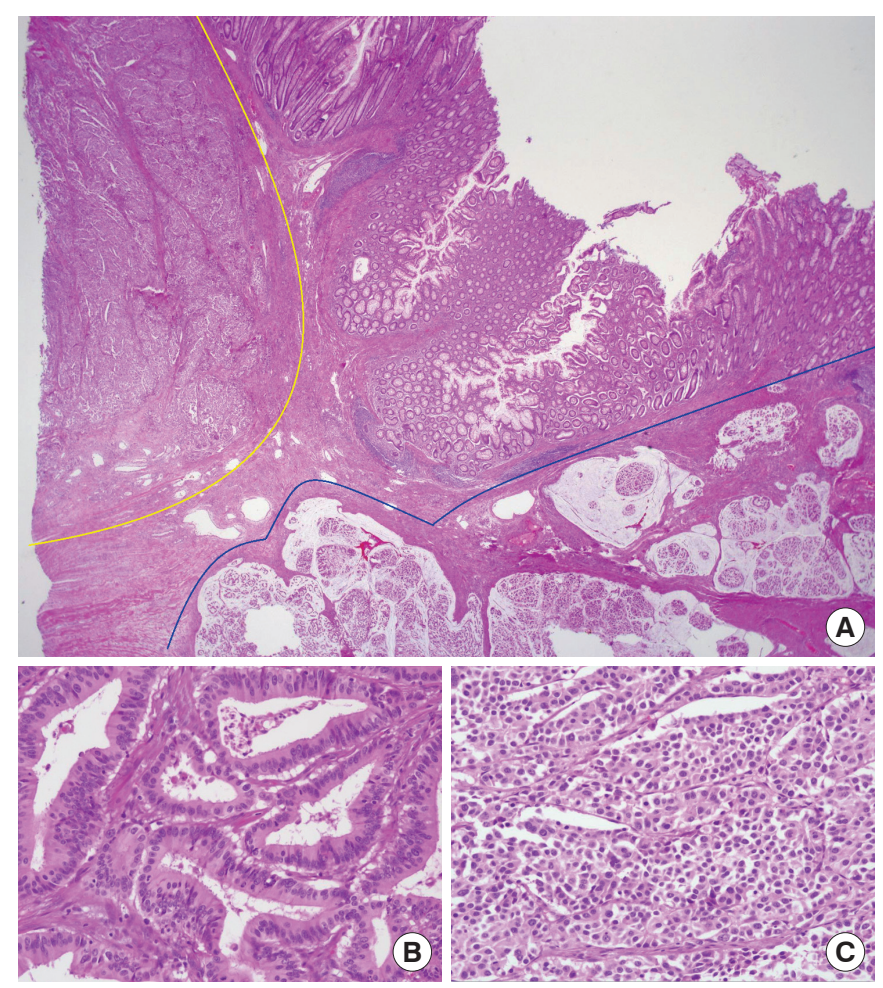

Fig. 4. (A) Hematoxylin and eosin stained sample at $\times 40$ magnification demonstrated sheets of monomorphic large cells with large round nuclei and abundant cytoplasm (inside the yellow line) and islands of tumor cells with glandular structures (inside the blue line). Hematoxylin and eosin stained tissue at $\times 200$ magnification was consistent with a poorly differentiated adenocarcinoma (B) and a neuroendocrine carcinoma (C).

resented at least $30 \%$ of the tumor [5]. Such neoplasms are now called "mixed adenoneuroendocrine carcinomas" (MANECs) according to the most recent WHO classification of gastrointestinal tract neoplasms [2].

A few studies of and some case reports on the rarely found MANEC tumor have been published. According to a recent review of endocrine cell carcinomas of the colon and rectum, most endocrine cell carcinomas of the colon and rectum are MANECs. Although the limited numbers of studies prevent our drawing generalized conclusions, the prognosis for patients with these tumors is poorer than it is for patients with routine colorectal cancer [6]. Hence, in most cases, the diagnosis should be confirmed solely by using a histologic study. MANECs containing a well-differentiated neuroendocrine tumor component and an adenocarcinoma component should be treated as adenocarcinomas. MANECs containing a poorly differentiated neuroendocrine carcinoma (NEC) component should be treated as NECs [7]. Patients with MANEC lesions containing a higher ratio of the adenocarcinoma component may have a better prognosis than patients with a lower ratio. However, circumspection is required for the interpretation of these results because the ratios of the adeno-

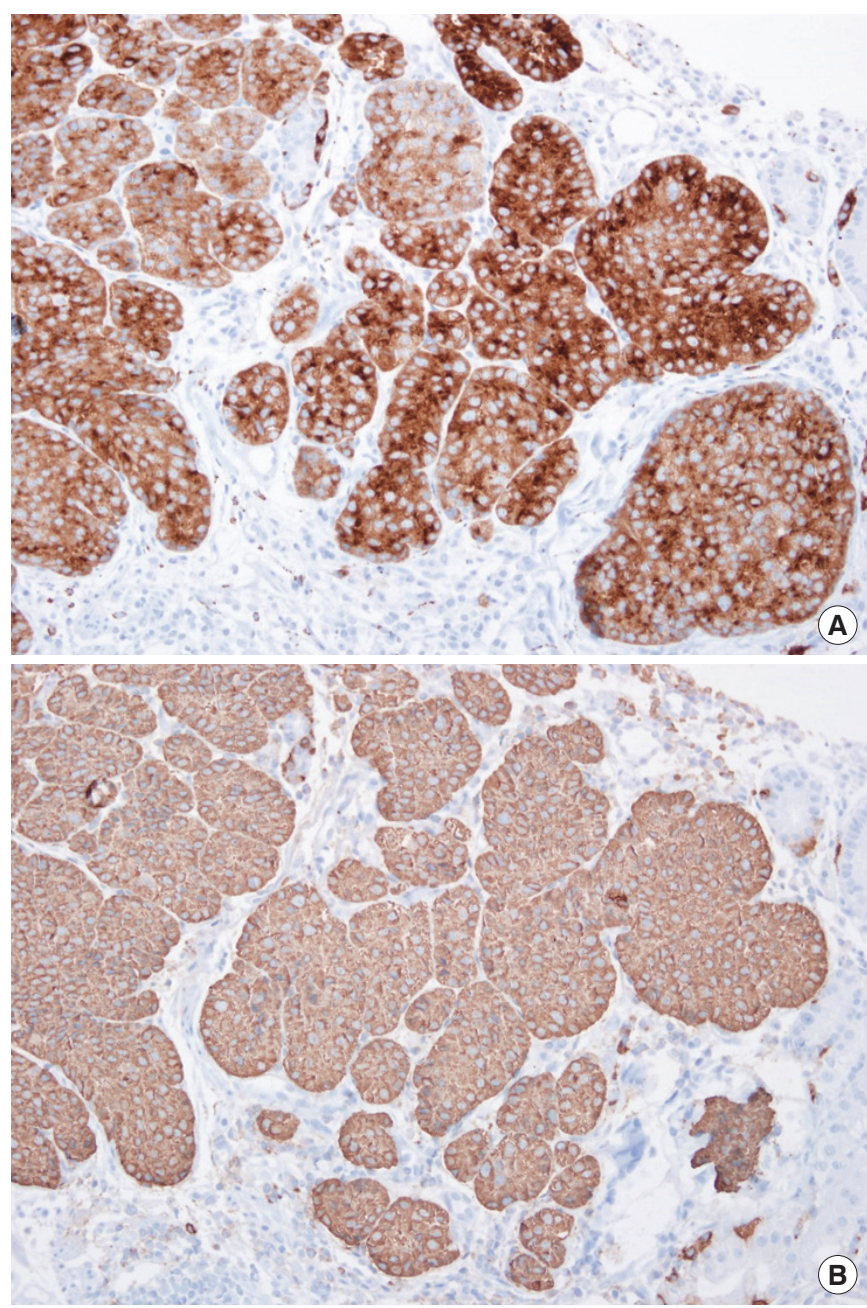

Fig. 5. (A) The tumor cells revealed a positive and diffuse reaction. Immunohistochemical staining for synaptophysin $(\times 200)$. (B) The tumor cell revealed a positive reaction in the neuroendocrine differentiation area. Immunohistochemical staining for chromogranin $(\times 200)$.

carcinoma component were obtained from only a small part of the whole tumor in the pathological specimen section.

The optimal management strategy for patients with a MANEC is largely unknown due to the rarity of these neoplasms. Generally, the more aggressive component of the MANEC should be taken into account when considering treatment. Adjuvant chemotherapy for patients with a MANEC should also be considered because some reports have indicated that it is effective [8]. However, which chemotherapy regimen is most effective in managing patients with a MANEC is not yet clear due to the small number of reports. Thus, there is no one best chemotherapy regimen for patients with a MANEC.

In conclusion, we reported a rare case of a patient with a MANEC, with particular emphasis on physician awareness of the term and the spectrum of MANECs, optimal treatment, and a better un- 
derstanding of tumor prognosis. Also, additional studies are required to define more conclusively the optimal management for patients with MANECs to achieve improved outcomes.

\section{CONFLICT OF INTEREST}

No potential conflict of interest relevant to this article was reported.

\section{REFERENCES}

1. La Rosa S, Marando A, Sessa F, Capella C. Mixed adenoneuroendocrine carcinomas (MANECs) of the gastrointestinal tract: an update. Cancers (Basel) 2012;4:11-30.

2. Rindi G, Arnold R, Bosman FT. Nomenclature and classification of neuroendocrine neoplasms of the digestive system. In: Bosman FT, Carneiro F, Hruban RH, Theise ND, et al., editors. WHO classification of tumors of the digestive system. Lyon: IARC Press; 2010:13-4.
3. Klappenbach RS, Kurman RJ, Sinclair CF, James LP. Composite carcinoma-carcinoid tumors of the gastrointestinal tract. A morphologic, histochemical, and immunocytochemical study. Am J Clin Pathol 1985;84:137-43.

4. Lewin K. Carcinoid tumors and the mixed (composite) glandular-endocrine cell carcinomas. Am J Surg Pathol 1987;11 Suppl 1:71-86.

5. Solcia E, Klöppel G, Sobin LH. Histological typing of endocrine tumours (WHO International Histological Classification of Tumours). 2nd ed. Berlin: Springer; 2000.

6. Komatsubara T, Koinuma K, Miyakura Y, Horie H, Morimoto M, Ito $\mathrm{H}$, et al. Endocrine cell carcinomas of the colon and rectum: a clinicopathological evaluation. Clin J Gastroenterol 2016;9:1-6.

7. Hervieu V, Scoazec JY. Mixed endocrine tumors. Ann Pathol 2005; 25:511-28.

8. Vanacker L, Smeets D, Hoorens A, Teugels E, Algaba R, Dehou MF, et al. Mixed adenoneuroendocrine carcinoma of the colon: molecular pathogenesis and treatment. Anticancer Res 2014;34: 5517-21. 